\title{
Avanzando en una formación de trabajadores/as sociales con perspectiva latinoamericana: Una experiencia de doble titulación
}

\section{Advancing in a training of social workers with latin american perspective: A double degree experience}

\author{
MARIBEL FLORIÁN BUITRAGO \\ Docente investigadora del Programa de Trabajo Social de la Universidad de La Salle. (Colombia) \\ maflorian@unisalle.edu.co / https://orcid.org/0000-0003-4361-0469
}

WENDY GODOY ORMAZÁBAL

Docente y Coordinadora Académica de la Escuela de Trabajo Social de la Universidad Católica Silva Henríquez, (Chile) wgodoy@ucsh.cl / https://orcid.org/0000-0002-9426-7878

\section{RUTH LIZANA IBACETA}

Directora de la Escuela de Trabajo Social de la Universidad Católica Silva Henríquez, (Chile) rlizana@ucsh.cl / https://orcid.org/0000-0002-0433-1689

\section{CARLOS MARIO RAMÍREZ RAVE}

Director del Programa de Trabajo Social de la Universidad de La Salle, (Colombia) cmramirez@lasalle.edu.co / https://orcid.org/0000-0003-3451-992X

\begin{abstract}
Resumen: El objetivo de este artículo es reflexionar en torno al proceso desarrollado por los equipos de dos Escuelas de Trabajo Social latinoamericanas - Universidad de La Salle, Colombia y Universidad Católica Silva Henríquez, Chile - en torno a construir una propuesta de formación que considerara la doble titulación, a partir de experiencias de movilidad estudiantil y de la lectura de un contexto compartido respecto de las demandas de la educación universitaria.

Cómo estrategia metodológica para el registro de la experiencia se utilizó la sistematización, justamente por los saldos pedagógicos que ofrece. La sistematización permite dar cuenta de las acciones y cambios realizados en distintos momentos del proceso, pero también causa la reflexividad y valoración de las acciones adelantadas.

Cabe señalar que este trabajo fue realizado en el marco de los procesos de internacionalización de la educación universitaria, cuestión que es hoy impulsada por distintos organismos nacionales e internacionales.
\end{abstract}

Palabras claves: Docencia Universitaria - Internacionalización -Movilidad - Doble Titulación- Trabajo Social 


\begin{abstract}
Summary: The objective of this article is to reflect on the process developed by the teams of two Latin American Social Work Schools - Universidad de La Salle, Colombia and Silva Henríquez Catholic University, Chile - around building a training proposal that will consider the double degree, based on experiences of student mobility and reading a shared context regarding the demands of university education. As a methodological strategy for the registration of experience, systematization was used, precisely because of the pedagogical balances it offers. The systematization allows to account for the actions and changes made at different moments of the process, but also causes the reflexivity and valuation of the advanced actions.

It should be noted that this work was carried out within the framework of the internationalization processes of university education, an issue that is today promoted by different national and international organizations.
\end{abstract}

Keywords:University Teaching - Internationalization -Mobility - Double Degree- Social Work

\section{PUNTO DE PARTIDA COMPATIENDO UNA LECTURA DEL CONTEXTO DE LA FORMACIÓN}

Los paradigmas que han sustentado la formación universitaria moderna entraron en crisis en las últimas décadas del siglo XX. Desde el encuentro de Bolonia (1999) las universidades han ido modificando los procesos de enseñanza y aprendizaje, al igual que sus modelos de gestión (Gairín et al, 2011) en pro de ajustar la formación universitaria a los cambios de un nuevo contexto.

El escenario del siglo XXI se caracteriza por la flexibilidad y complejidad en diversas dimensiones de la vida cotidiana; constituyéndose en un desafío para los procesos de formación; sobre todo, porque se requiere de una formación cada vez más dinámica, que permita incorporar rápidamente los cambios en sus aulas (Bauman, 2004). Es así como se puede señalar que la educación superior debe enfrentar un nuevo contexto, representado por: la mundialización del mercado laboral, que incide en que no sólo se debe dar respuesta a necesidades de formación locales, sino que también incorporar aspectos regionales y globales involucrados en el mercado de trabajo y en el desarrollo de las ciencias, tal como señalan Brunner et al (2016: 17)

El mundo de la educación superior vive un tiempo de convergencia a nivel global de los problemas y desafíos, al mismo tiempo que su abordaje y las soluciones diseñadas se caracterizan por tener un alcance que algunos autores llaman glonacal; es decir, a la vez global, nacional y local, con una fase de convergencias intermedias también en los espacios regionales.

Otro elemento es la definición de una sociedad del conocimiento, donde la tecnología tiende a considerarse como eje de un "mayor impacto para el desarrollo económico y social de las comunidades" (Mora, 2004: 21)

La revolución científico-tecnológica y el desarrollo de internet constituyen, sin duda, uno de los mayores fenómenos culturales del siglo. Sustentados en la cibercultura global -conectada e instantánea-, los avances tecnológicos fomentan innovaciones importantes en la transmisión de la información, lo cual repercute, sin duda, en la cantidad y velocidad de producción del conocimiento. De igual modo, las transformaciones tecnológicas modifican también las modalidades tradicionales del trabajo académico a través de mecanismos tales como teleconferencias, correo electrónico, coloquios vía internet, revistas en red, etcétera, posibilitando que maestros e investigadores puedan elegir interlocutores afines en cualquier parte del mundo (Waldman; 2000:231).

Un tercer elemento refiere a la masificación de la formación universitaria, lo que es rescatado por Brunner, J. \& Miranda, A. (2016: 17) quienes señalan que:

La masificación y progresiva universalización del acceso transforma los sistemas nacionales que durante siglos han atendido a minorías en sistemas abiertos que buscan ofrecer oportunidades de estudio y experiencia formativa par toda la población de jóvenes y, crecientemente, además, de adultos.

Estos aspectos pueden reconocerse, también, en las metas 2021 propuestas en la Cumbre Iberoamericana de Jefes de Estado, en torno a la educación (2010), donde entre otros se propone promover la cooperación orientada a la mejora 
continua de la calidad de la educación superior. Lo anterior se constituye en una oportunidad de pensar en un trabajo colaborativo, como una contribución en el contexto latinoamericano, a partir de la generación de redes académicas que nos permitan pensar un trabajo social más allá de las fronteras nacionales. Asimismo, la Unesco señala la importancia de hacer "circular experiencias de movilidad estudiantil y académica y auspiciar una reflexión sobre sus implicaciones en el diseño y operación de programas." (Unesco: 2014)

Suscribir la experiencia de internacionalización, más allá de las fronteras de la movilidad, de manera que implique responder a los desafíos que se presentan en este escenario de complejización de la formación universitaria, es uno de los desafíos que nos propusimos al pensar este proyecto. Lo pensamos como una práctica de innovación, que ha significado movilizar dos institucionalidades, más allá de las propias carreras. (Aupetit, 2018)

Es por ello que la lectura anterior necesariamente interpela a las universidades en la búsqueda de nuevas estrategias curriculares y didácticas que favorezcan "la constante revisión curricular de la enseñanza y el impulso de novedosas titulaciones (dobles y conjuntas)" (Brunner, J. \& Miranda, A. 2016:29), cuyo centro del aprendizaje sea el estudiante, reconociéndolo como un agente activo y responsable de su proceso formativo a lo largo de la vida, valorando "que cada persona aprende de una manera específica y conocemos que cuanto más individualizamos la enseñanza -es decir, más la ajustamos a las características del alumno- conseguimos mejor aprendizaje" (Roca, 2010:95).

En concordancia con los planteamientos anteriores, es que ambas Escuelas inician un proceso de rediseño del currículo que, en el caso de la UCSH, se materializa en la búsqueda de una formación con identidad, con calidad y con responsabilidad, por medio de una pedagogía en un ambiente universitario comunitario, que privilegia la mediación del conocimiento, que promueve la responsabilidad ética, civil y la formación sociopolítica. A su vez, incorpora las dimensiones, del saber ser, conocer, hacer y relacionarse; asumiendo a los estudiantes como el centro del proceso, articulado su aprendizaje al proyecto de vida personal, a la búsqueda de una sociedad más justa y democrática, que promueva la igualdad y justicia (UCSH; 2015).

Para ambos programas de Trabajo Social, además de la pertinencia curricular, pensar la doble titulación se constituye en una estrategia de articulación a las dinámicas mundiales de la educación que le permiten tener una pertinencia regional y globalizada en la oferta académica, logrando que los y las estudiantes puedan incorporar conocimientos con una capacidad de actuar local y globalmente en una relación bidireccional que permita reconocer las situaciones que se viven en el país, desde una comprensión global y asociada a fenómenos latinoamericanos y mundiales de diverso orden (político, económico, social, cultural, entre otros) lo que permite una intervención pertinente y generadora de impactos.

Tampoco estuvo ausente, pensar esta posibilidad desde la idea de generar oportunidades a estudiantes, que, en su gran mayoría, para ambas instituciones, son la primera generación en sus familias que accede a la universidad, cumpliendo así propósitos que Aupetit, S. (2018) denomina redistribución democrática de oportunidades.

Para lograr lo anterior, la Universidad de la Salle, ha construido un horizonte programático y estratégico donde el relacionamiento internacional, se constituye en un referente de sus programas académicos. El programa de trabajo social ha sido uno de los programas pioneros en interpretar este direccionamiento, por este motivo ha incurrido en acciones como la acreditación del programa con el Sistema Latinoamericano SILEU promovido por CLACSO como una de las redes de carácter socio crítico en ciencias sociales más representativas en Iberoamérica y Latinoamérica, ha gestado convenios de diverso orden con entidades Latinoamericanas, ha incentivado la movilidad académica de sus estudiantes a universidades en todo el mundo siendo Argentina, Chile, México y España en su orden los países hacia donde más se ha activado la movilidad, promoviendo al mismo tiempo agendas de investigación con otros países. En tal sentido la doble titulación, materializa estas iniciativas y les da un gran sentido estratégico de posicionamiento en el pensamiento y actuación global y latinoamericana, legitimando lo que ha construido en los últimos años, la oportunidad de ser un programa abierto al mundo, legitimando la acreditación que actualmente tiene en CLACSO.

Por su parte, Trabajo Social de la UCSH, igualmente, ha considerado dentro de su horizonte estratégico y, respondiendo a lineamientos institucionales respecto de internacionalización y movilidad académica, la permanente preocupación por la calidad de sus programas de formación, siendo la acreditación de la carrera de pre grado, una de sus fortalezas. Asimismo, con el apoyo institucional correspondiente, ha promovido el intercambio estudiantil y académico, siendo los países de España, Brasil, Argentina y Colombia los lugares más recorridos por nuestras y nuestros estudiantes. Como Escuela participa activamente en redes de formación e investigación nacionales e internacionales, siendo un esfuerzo no menor dar visibilidad a su producción académica, a través de la publicación en editoriales de gran prestigio a nivel del país y de América Latina. Cuenta con una Revista de circulación 
periódica hace más de 25 años, indexada en el directorio y catálogo LATINDEX 2.0. La doble titulación se constituye así en una puerta de llegada, en tanto brindar opciones de movilidad profesional a nuestros egresados y de partida en el sentido de pensar (se) desde América Latina la formación de trabajadoras y trabajadores sociales.

Dar cuenta de esta experiencia busca aportar a la discusión acerca de la internacionalización de la educación superior, pensando la doble titulación como una oportunidad de innovación, no sólo para una carrera específica, sino para la propia institucionalidad universitaria, reconociendo que la movilidad, el intercambio y la doble titulación, aparece como un fenómeno interesante de ser estudiado, tal como lo vienen realizando otros autores, a partir de los desafíos propuestos por la Unesco.

Este esfuerzo por sistematizar esta experiencia, surge por la necesidad de conocer, darse a conocer y cualificar las propias prácticas, (Ruiz, 2001) en el ámbito académico.

Asumimos lo señalado por Oscar Jara (2011) para quien la sistematización no son solo datos que se recogen y ordenan, sino que busca obtener aprendizajes críticos de las experiencias, por ello, cuando este autor habla de sistematización, lo hace denominándola "sistematización de experiencia" (Jara, 2011). Desde esta visión buscamos ir más allá, mirando la experiencia como un conjunto de procesos históricos, complejos en los cuales intervienen distintos actores y que realizamos en un contexto determinado y un momento institucional del cual se forma parte.

\section{FUNDAMENTOS DEL PLAN DE FORMACIÓN DE TRABAJO SOCIAL UCSH}

En este acápite se asume la reflexión del equipo de la Escuela de Trabajo Social de la UCSH, la que está contenida en diversos documentos de circulación interna. Reconocemos que la emergencia del Estado neoliberal, marca un nuevo tipo de relaciones entre la intervención social y la nueva cuestión social. Esta se caracteriza por la creciente exclusión social y expulsión de personas de sus territorios y la fragmentación de una sociedad sometida a los intereses del mercado (Rozas, 2004). Ello plantea enormes desafíos a la disciplina del trabajo social, en el sentido de desmitificar la naturalización de la cuestión social en el actual escenario de las relaciones sociales, reposicionando a nivel teórico, político y ético el lugar público de lo social. Esa despolitización es la que está a la base del desmantelamiento de los servicios públicos, de las políticas sociales, de la destitución de los derechos sociales y de la privatización y refilantropización en el abordaje de la cuestión social; preocupaciones que buscan ser asumidas en ambas propuestas curriculares.

La intervención en lo social se configura como objeto de la disciplina y de la profesión de dos maneras distintas y complementarias. Como objeto, en tanto lo que se busca es la producción de conocimientos sobre lo social directamente ligados a la acción y a la intervención profesional sobre algún campo específico a través del cual lo social se manifiesta. El propósito disciplinar sería, en consecuencia, acumular conocimiento fundamentado sobre cómo actuar o cómo no actuar frente a la especificidad y complejidad de las distintas manifestaciones de la cuestión social, sirviendo la investigación a identificar la particularidad que debiese poseer la acción profesional en función de ello. Mientras, el objeto de la profesión serán situaciones de intervención que se configuran a través de la vivencia, por parte de individuos y/o grupos, de problemas sociales diversos. El propósito de esta intervención sería el de co-construir con los sujetos afectados alternativas que permitan mejorar las condiciones que se hayan constituido como problema. Adicionalmente, acompañar los procesos de constitución de ciudadanías activas con capacidad de agencia e interpelación en relación con la garantía de derechos.

Para que exista retroalimentación efectiva entre estos dos campos, la intervención debe ser registrada y sometida a evaluación permanente estimando su capacidad para producir cambios, teniendo en consideración todos los factores que afectan dicho propósito. En ambos casos, el resultado debieran ser hipótesis explicativas y/o interpretativas orientadas hacia la profundización de la comprensión sobre la problemática, siempre y necesariamente, en directa relación con la pregunta acerca de cómo actuar sobre ella.

La segunda premisa relativa al Trabajo Social que anima esta reflexión y que estaría estrechamente vinculada a la primera, es que en este marco de discusión el referente orientador de la acción, son los valores y la intencionalidad política del Trabajo Social. Estos valores se relacionan con el desarrollo histórico de la profesión y que podríamos resumir en el gran propósito de contribuir a la emancipación humana. En tanto, la dimensión política estaría dada por la intención de favorecer la redistribución del poder al interior de la sociedad y contribuir a la constitución de comunidades políticas y de sujetos/ciudadanos, tal como lo muestra el trabajo de Travi (2014) sobre las pioneras del Trabajo Social. Ambas aspiraciones suponen importantes desarrollos argumentales desde la ética y la filosofía política, así como también una discusión más profunda al interior del colectivo profesional respecto de cuáles serían los principios de legitimación teóricos y éticos 
de esta "emancipación", entendiendo que dichos principios han de inscribirse en la crisis de una teoría crítica post moderna a la manera en que lo encontramos en Salas (2008) parafraseando a de Sousa Santos.

No existe un principio único de transformación social. Asimismo no existen agentes históricos ni tampoco una forma única de dominación. Los rostros de la dominación y de la opresión son múltiples, también deben ser diversas las formas y los agentes de resistencia a ellos. Más que una teoría común, lo que se requiere es una teoría de la traducción capaz de hacer mutuamente inteligibles las diferentes luchas, permitiendo de esta manera que los actores colectivos se expresen sobre las opresiones a las que hacen resistencia y las aspiraciones que los movilizan. (Salas, 2008:417).

Asumir la intencionalidad ético política de la profesión, más allá de la definición que justifique los principios que la sostienen, supondrá que el conocimiento y la intervención en la realidad social queden supeditados a dicha intencionalidad y que ello significará para el profesional, el desarrollo de habilidades de negociación, mediación, coordinación y articulación eficiente de puntos de vista en conflicto (Schön, 2010). Junto con ello, deberá ejercitar la reflexión crítica en torno a las situaciones en las que interviene y habilidades para ensayar distintas estrategias para la reformulación de las interpretaciones que se van construyendo del fenómeno que se aborda (flexibilidad), convergiendo de manera dinámica, los conocimientos adquiridos en la formación académica y el contexto de intervención, institucional, comunitario u otro.

Las aspiraciones aquí mencionadas han permeado el trabajo de reflexión, diseño y materialización de los planes de estudios de ambas escuelas. La convergencia que existe en relación con el paradigma socio-critico, como fundamento en la labor pedagógica, ilustra la preocupación ética y política que ambas escuelas tienen en relación con la construcción de lo social. A ello suma la convergencia de competencias formativas, no necesariamente visibles en la denominación de los espacios académicos, pero sí en el ideario de formación integral que ambas escuelas proponen.

\section{LA DOBLE TITULACIÓN. PENSAR UN TRABAJO SOCIAL MÁS ALLÁ DE NUESTRAS FRONTERAS}

Como se ha señalado anteriormente, en un contexto de una educación universitaria globalizada, las universidades buscan fortalecer vínculos de colaboración internacionales, que favorezca la producción académica, el intercambio de docentes y estudiantes (UCSH, 2018).

Respondiendo a esta búsqueda, es que las escuelas de trabajo social de la UCSH y La Salle inician el proceso de mirar un plan de estudios, que permita la movilidad estudiantil y académica, desde un marco reflexivo que integra a los equipos en diseños de una propuesta formativa que recoja el contexto latinoamericano, sus desafíos, tensiones y oportunidades.

Algunas de las preguntas que ambas Escuelas intentamos responder, durante este proceso y que fueron compartidas en encuentros, reuniones y trabajo en equipo son: ¿Desde qué marcos epistemológicos y teóricos se pueden comprender en el trabajo social, la sociedad, las culturas, los sujetos y sus múltiples relaciones y determinaciones? ¿Cuáles son los elementos que posibilitan la fundamentación teórica y metodológica del Trabajo Social para dar respuestas a los problemas y contextos actuales? ¿Cómo establecer procesos de intervención profesional basados en la ética profesional, el respeto por los derechos humanos y la justicia social? ¿Cómo avanzar en el diálogo con otras disciplinas y saberes que permitan construir propuestas interdisciplinares, transdisciplinares de investigación e intervención que den respuesta a la complejidad de la realidad social y las problemáticas actuales? Preguntas que son recogidas desde las propuestas curriculares.

Es así como en la búsqueda por aportar, desde una perspectiva latinoamericana al desarrollo de la profesión, se valoró altamente positivo avanzar en un acuerdo de trabajo que permitiera favorecer la formación de trabajares/as sociales desde ejes comunes: a saber:

3.1 Una identidad institucional compartida, que busca promover la dignidad y el desarrollo integral de las personas.

3.2 Una formación culturalmente situada, que reconoce y valora la diversidad.

3.3 Una impronta ética profesional, basada en el reconocimiento de los derechos humanos y la justicia social.

3.4 Una propuesta que busca contribuir al desarrollo integral de sus estudiantes, a través de un proyecto de formación universitaria con identidad, calidad y responsabilidad compartido.

Una especial consideración implicó el reconocimiento de planes de formación general, con claro sello identitario en ambas instituciones, tal como lo muestra la gráfica siguiente: 


\section{GRÁFICA 1}

\section{LAS SLLLE}

BLOQUE: FORMACIÓN LASALLISTA - FORMACIÓN INTEGRAL

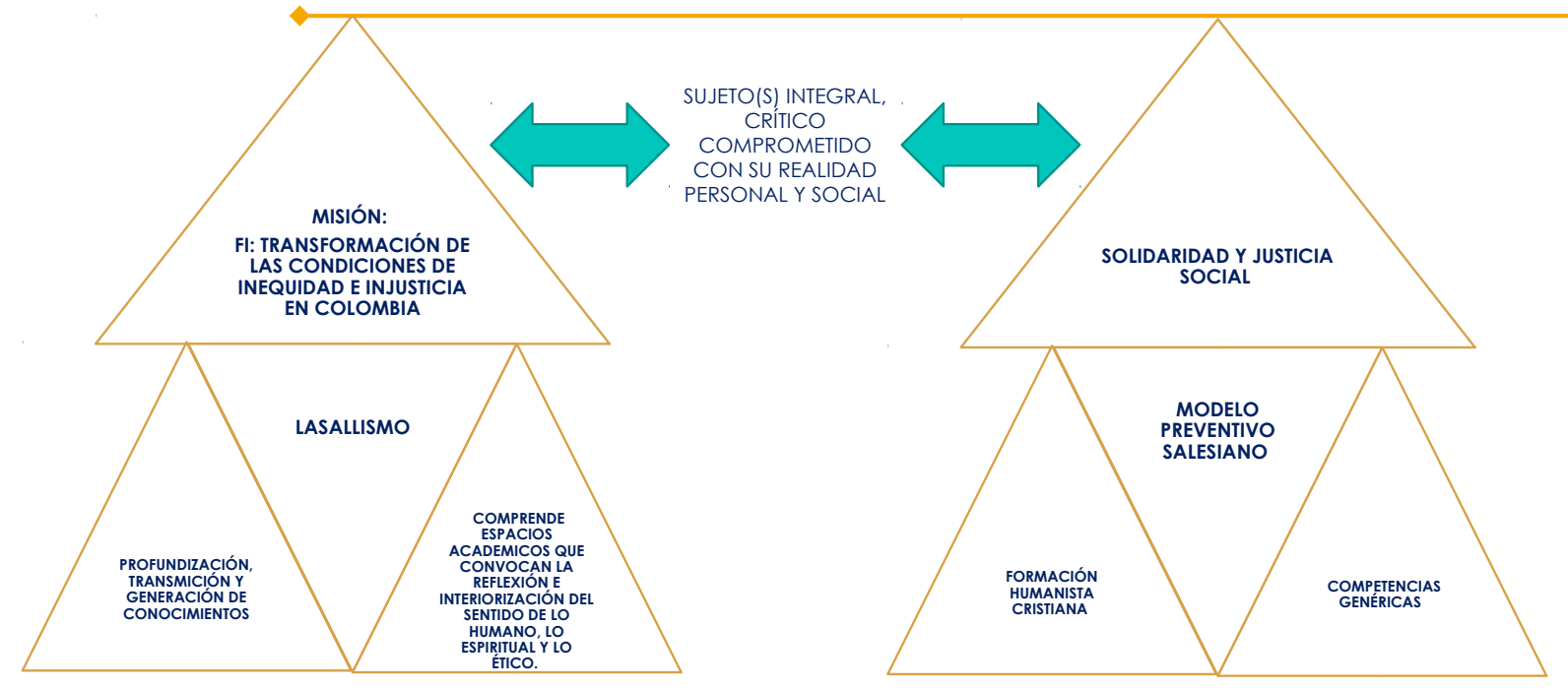

Fuente: elaboración del equipo de trabajo

La firma de un acuerdo de doble titulación fue producto de un proceso, que buscó responder inquietudes académicas, desde un ethos de formación profesional, que releva una concepción de sujeto integral, crítico y comprometido con su realidad personal y social, ubicándolo en el centro del quehacer universitario; que comparte una misión vinculada a la transformación de las condiciones de inequidad e injusticia y propone la solidaridad y la justicia social para lograrlo, a partir de la pedagogía Lasallista y Salesiana.

\section{LA DOBLE TITULACIÓN. FRUTO DE UN PROCESO DE TRABAJO CONJUNTO}

\subsection{Construyendo vínculos}

En el año 2011 ambas Universidades firman convenio amplio que potencia que nuestras Escuelas nos interesemos por iniciar una búsqueda de espacios posibles de ser compartidos. Desde allí se diseña un plan de trabajo de corto y mediano plazo que implica el intercambio de académicos y estudiantes, la participación en encuentros, pasantías, foros, seminarios, clases magistrales, y la elaboración de artículos que nutren la publicación en las revistas, que en ese momento ambas escuelas sostenían.

En el cuadro 1 se nos muestra el proceso realizado.
Luego de cuatro años de trabajo conjunto, se firma Convenio de Colaboración Académica para el otorgamiento de Doble Titulación. Lo anterior significó ubicar este convenio dentro del contexto institucional, involucrando a las Facultades y Unidades de gestión académica y jurídica para su logro, ya que implicó resguardar temas de calidad y de procedimientos legales que avalen dicho proceso.

Sobre esta historia de relacionamiento institucional es clave destacar algunos puntos comunes que se fueron evidenciando en las actividades que se desarrollaron en cuatro años de un convenio con altos niveles de vitalidad, algunos de los elementos más destacados de convergencia institucional son los siguientes:

- Concepción interdisciplinaria de la formación profesional: La inscripción de los programas de ambas universidades en facultades de Ciencias Sociales, Jurídicas y Económicas; en el caso de la UCSH y una Facultad de Ciencias Económicas en el caso de la Universidad de la Salle, sumado a otras dinámicas investigativas y formativas, nos ha implicado una postura de formación interdisciplinaria abierta al mundo y sus necesidades que ha sido clave para gestar una relación de apertura que permite tejer relaciones académicas, investigativas y de conocimiento, lo cual fue clave en el proceso. 


\section{CUAdro 1}

Confianza: Construcción de posibilidad

2011 Convenio Marco. Mayo 28 y 30 de 2013

Realización de una agenda común a nivel de Facultades: Revista, Movilidad, Investigación y Docencia.

2014 Movilidad Docente (prácticas y currículo)

\section{Abril 27,28 y 29 de 2015}

Visita académica, decano, docentes y directora del programa del TS: rediseño curricular.

\section{Octubre 24 y 25 de 2016}

Visita académica Directora y coordinadora académica del Programa de Trabajo Social de la Universidad Silva Henríquez

Proceso doble titulación.

\section{Apuesta Pedagógica: por qué y para} qué de un currículum transnacional

\section{Febrero 2017}

Diseño de la Ruta Metodológica para poner en diálogo las mallas de ambos programas: créditos, denominaciones e intencionalidades de espacios académicos y competencias.

\section{Marzo - agosto 2017}

Diálogos y construcciones académicas con coordinación de currículo.

\section{Administración: convenio y organización para el funcionamiento de la propuesta de movilidad académica.}

Doble Titulación en Trabajo Social.

Fuente: elaboración del equipo de trabajo

- Articulación de una concepción pedagógica flexible, abierta a la lectura de contexto y altamente reflexiva de las realidades globales y latinoamericanas: con un currículo vivo, donde los docentes investigan y realizan procesos de proyección social del conocimiento que luego regresan a las aulas convertidos en materias electivas y contenidos didácticos que enriquecen la formación y la hacen más pertinente e innovadora.

- Articulación de los componentes de investigación e intervención trasversal en la mirada curricular: este elemento ha sido clave en ambos programas, para una formación de los estudiantes capaz de indagar por las razones y condiciones generadoras de los fenómenos y problemas sociales para generar trasformaciones $\mathrm{e}$ impactos posibles a través de una práctica e intervención constante que no solo está inscrita a un momento de la formación, lo que permite la consolidación de sujetos reflexivos, críticos y comprometidos con una acción política innovadora y constante en los territorios.

- Relacionamiento permanente con los gremios académicos y profesionales como elemento común que incide en unos programas con una mirada ética y política de la formación: lo que en ambas escuelas convierte a nuestros docentes y directivos en participantes activos de estos espacios gremiales, apostando en ellos a un trabajo social latinoamericano y pertinente revertiéndose en una mirada y apuesta formativa en los programas.
Desde el año 2018 se potencia la movilidad estudiantil en la modalidad de doble titulación, pudiendo dos estudiantes de la UCSH, cursar un semestre en La Salle, y una estudiante de La Salle, un semestre en la UCSH. Al respecto, algunos de los saldos pedagógicos connotan tanto los aprendizajes académicos como personales:

Ha sido una experiencia maravillosa de aprendizaje permanente, muy recomendable. Hemos aprendido mucho de la cultura, de la carrera de la manera cómo se trabaja (Testimonio estudiante UCSH, 2018).

Haber tenido la oportunidad de hacer un intercambio en la UCSH, me permitió conocer más sobre los procesos sociales, políticos, económicos y culturales Latinoamericanos que son ejes transversales de nuestro continente, algunos de ellos incluso similares al caso colombiano. Destaco de igual manera, la riqueza cultural y social de esta experiencia, aprendí mucho de historia latinoamericana y de herramientas complementarias para el abordaje de nuestra profesión (Testimonio estudiante ULSA, 2018).

\section{MÉTODO DE TRABAJO PARA EL LOGRO DE LA DOBLE TITULACIÓN}

Integrando la sistematización cómo parte de la estrategia metodológica, se diseñó un plan de trabajo inicial, que entre sus puntos consideraba como intencionalidad favorecer condiciones que permitieran arribar a una doble titulación para estudiantes de la carrera de trabajo social de ambas universidades. 


\section{CUAdRo 2}

A nivel de Gestión:

Revisión documental: Políticas Nacionales e Internacionales en torno al tema

Firma de Convenio Marco General entre las Universidades.

Análisis de las potencialidades de este convenio, con autoridades.

Coordinación Interna a Nivel de Universidad y Facultad.

Reuniones de trabajo entre Unidades Académicas, Facultades involucradas, equipos de relaciones internacionales y de gestión académico curricular.

Elaboración y firma de convenios específicos, orientados al logro de productos académicos específicos.
A nivel Curricular

Construcción de Agendas con acuerdos preliminares, respecto de los planes de estudio:

Análisis de Modelo de Formación de ambas Universidades.

Rediseño de Planes de Estudios, elaborados de manera colaborativa

\section{Análisis desde el Sistema de Créditos Transferibles}

Elaboración de Planes de Equivalencia

Propuesta específica de convenio de doble titulación: requisitos exigibles a los estudiantes de ambas universidades;

Evaluaciones de procesos y ajuste de agenda de trabajo.

Fuente: elaboración del equipo de trabajo

Como registro del proceso se implementó un sistema registro visual (fotografías) y escrito, a través de actas con los acuerdos de trabajo. Estos documentos fueron firmados por los participantes directos y ratificados por los decanos de las facultades correspondientes.

Esta modalidad permitió mantener un monitoreo permanente de los avances y de las modificaciones introducidas, apoyada además por el uso de las TICS.

Las principales acciones desarrolladas en esta línea se sintetizan en el cuadro 2.

Previo a la implementación se logran los acuerdos que permiten viabilizar la doble titulación, en el entendido que se trabaja desde miradas institucionales afines, planes de estudios equivalentes, que hacen posible que, un/a estudiante, cursando un número de créditos determinados, en un semestre, pueda acceder a ella.

\section{RUTA METODOLÓGICA}

Siguiendo la lógica de la sistematización, la ruta metodológica desarrollada, implicó la revisión in extenso de los planes de estudios de ambas escuelas, elaborando matrices de correspondencia académica, para cada una de las actividades curriculares, a partir de los perfiles de egreso declarados. En paralelo, en ambas instituciones se estableció un proceso de re diseño curricular, que facilitó la convergencia de ambas mallas curriculares, tal como se aprecia en la gráfica 2 .

Como resultado de esta fase de trabajo, se determinan los requisitos para que los estudiantes con movilidad estudiantil, que opten a doble titulación, puedan recibirla. En la gráfica 3 se sintetizan estos requerimientos.
Finalmente, un/a estudiante de trabajo social de las universidades comprometidas en este convenio, una vez finalizado su plan de estudios en la universidad de origen y, habiendo aprobado los créditos exigidos por la universidad de destino, logra obtener la doble titulación.

\section{IMPLEMENTACIÓN DEL CONVENIO DE DOBLE TITULACIÓN}

La fase de implementación del convenio de doble titulación ha implicado nuevos desafíos relacionados con:

7.1 La difusión de la propuesta entre los y las estudiantes, aspecto que ha significado socializar los requisitos para postular, así como también los recursos económicos implicados en este proceso.

7.1.1. La coordinación permanente con diversas unidades al interior de cada universidad, relacionadas con la gestión académica - curricular para resguardar la validez académica de los procesos.

7.1.2. La coordinación entre ambas escuelas de Trabajo Social involucradas, para ajustar las cargas académicas de los/as estudiantes participantes, de manera de resguardad los procesos de titulación en sus universidades de origen.

7.1.3. El acompañamiento académico y de inserción de los/as estudiantes pasantes, tanto en la asignación de las actividades curriculares como de otros espacios formativos que ofrece la universidad, así como también de otros recursos de apoyo para el éxito de su proceso de formación. 
GRÁFICA 2

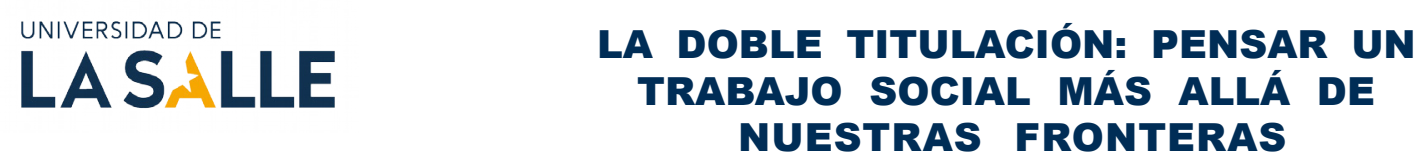

MALLA TRABAJO SOCIAL UCSH

METODOLÓGICA

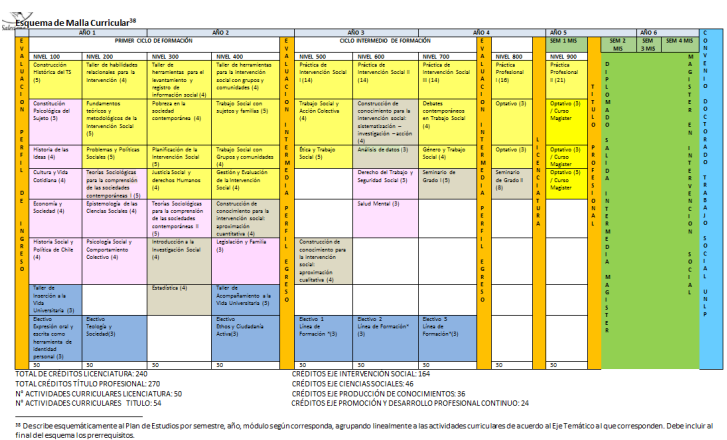

MALLA TRABAJO SOCIAL UNISALLE

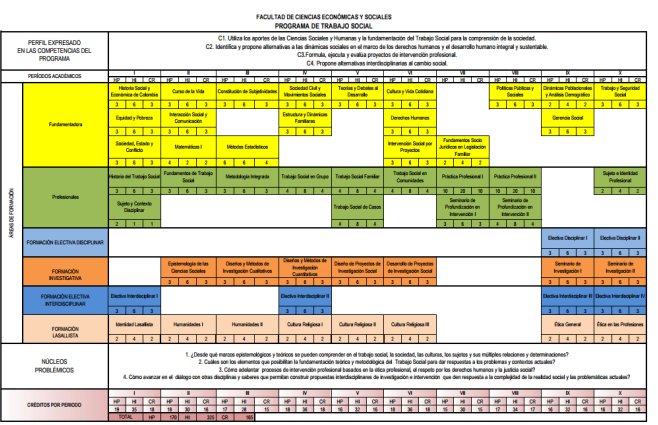

Fuente: elaboración del equipo de trabajo

GrÁFICA 3

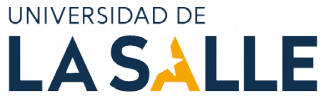

CONVENIO DE DOBLE TITULACIÓN EN TRABAJO SOCIAL, NOVIEMBRE 22 DE 2017

\section{CRÉDITOS A CURSAR}

Estudiantes de Unisalle en UCSH

El(la) estudiante de la UNISALLE deberá inscribir un mínimo de $\mathbf{3 0}$ créditos en la Carrera de Trabajo Social de la UCSH, es importante destacar que tres (3) de estos créditos corresponden al Plan de Formación Integral.

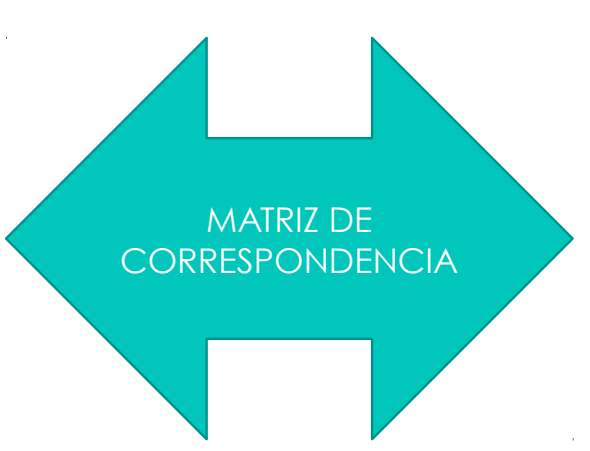

Estudiantes de UCSH en Unisalle

El(la) estudiante de la Unisilva deberá inscribir un mínimo de 16 créditos en la Carrera de Trabajo Social de la Unisalle, de los cuales son de carácter obligatorio los 4 correspondientes a los espacios académicos:

Identidad Lasallista

Cultura religiosa- - Jesús de Nazaret maestro de humanidad.

Fuente: elaboración del equipo de trabajo 


\section{APRENDIZAJES DEL PROCESO}

8.1 A nivel de la Gestión:

8.1.1. Reconocer la necesidad de instalar procesos flexibles, que permitan diseñar estrategias de largo plazo, en lo curricular, que favorezcan planes de formación académicos internacionales.

8.1.2. Contar con apoyos institucionales potenciadores de la innovación facilitó lograr acuerdos de esta naturaleza. En particular en este caso, dada la complejidad del proceso se evidencia la necesidad de incorporar equipos de otras unidades de apoyo a la gestión académica, tales como direcciones de internacionalización, jurídicas, académico/curriculares y financieras. Vital es el apoyo desde la decanatura, quien incorpora dentro de nuestras metas este logro.

8.1.3. Fortalecer el trabajo en equipo, que implica la generación de confianzas entre los equipos interinstitucionales involucrados, directamente en la reflexión y diseño de estrategias innovadoras, que recogen los desafíos de la formación universitaria del siglo XXI.

8.2 A nivel curricular:

8.2.1 Contar con equipos que tengan una comprensión local y global del proceso de formación, que, siendo capaces de recoger las particularidades, las inscriban en propuestas con incidencia regional.

8.2.2 Contar con equipos formados en diseño curricular, en este caso basado en competencias con sistema de créditos transferibles, modelo al que adscribe ambas universidades implicadas en este proceso.

8.2.3 Capacidad para consensuar perspectivas, criterios y enfoques de trabajo social, que permitan la generación de acuerdos y la materialización de ellos en una propuesta curricular equivalente. Ello implicó contar con la posibilidad de discutir perspectivas sobre la profesión y la disciplina que enriqueció los debates al interior de los equipos académicos.

8.3. A nivel de trabajo de los equipos de las escuelas:

8.3.1 Situar la Internacionalización como parte de la tarea pedagógica, que ya en el aula se encuentran estudiantes con experiencias formativas distintas.
8.3.2 Fortalecer el uso de diversas estrategias y metodologías, incorporando las Tics, en el quehacer académico.

8.3.3. A partir de esta experiencia se han desarrollado, otras acciones de colaboración entre las escuelas, tales como intercambios de docentes y clases magistrales, que han fortalecido la docencia, integrando la internacionalización en el aula.

\section{CONCLUSIONES}

La internacionalización se constituye actualmente en un imperativo para las instituciones de educación superior; ejemplo de ello son las diversas experiencias que están visibilizadas, desde la propia academia, que Unesco reconoce, a través de seminarios, encuentros y publicaciones, en torno a la temática.

Esta experiencia de doble titulación se inscribe en el campo de internacionalización, a través de la movilidad estudiantil, exigiendo a las instituciones universitarias, nuevos marcos de gestión institucional que recojan los desafíos de un mundo globalizado.

En el camino de construcción de un proyecto compartido, nos reconocemos con una visión próxima acerca de la formación universitaria del trabajo social, desde países que vivimos un mismo modelo de desarrollo, que tensiona la intervención social y desafía las propuestas de formación de trabajadoras y trabajadores sociales en América Latina.

Producto de estos años de trabajo es posible concluir que el interés de mirar la formación, más allá de las fronteras nacionales, ha sido un desafío para las Escuelas de Trabajo Social de la UCSH y UNISALLE. Ambos programas hemos buscado formar profesionales que puedan realizar intervenciones situadas en contextos de complejidad, aportando significativamente a la transformación de estos escenarios sociales.

Reconocemos también como eje común los esfuerzos por generar nuevos modelos de intervención y comprensión de la realidad social que permitan el mejoramiento de las condiciones sociales para el desarrollo humano de las personas y comunidades con las cuales trabajamos.

El sello de ambas propuestas curriculares, se inscribe en develar las desigualdades y proponer la justicia social, la responsabilidad ciudadana y la excelencia académica como horizontes en la formación de trabajadores sociales y profesionales de la intervención social a nivel latinoamericano.

Lo anterior nos ha planteado permanentes desafíos, en el sentido de desmitificar la naturalización de la cuestión social en el actual escenario de las relaciones sociales de desigual- 
dad que ha caracterizado al continente latinoamericano, reposicionando desde una perspectiva teórico - critica, política y ética el lugar público de lo social.

Enriquecer nuestras propuestas de formación e impulsar la movilidad de académicos, estudiantes y también de aquellos profesionales de gestión, permitió avanzar en la consolidación de la propuesta formativa de las escuelas, con calidad, identidad y responsabilidad, compartiendo un ideario de formación en torno al trabajo social, para poner en discusión en el concierto nacional y latinoamericano. Una experiencia como la descrita apuesta por la producción de un pensamiento colectivo en torno a la formación de trabajadoras y trabajadores sociales, que se tradujo en propuestas curriculares, equivalentes para ambas carreras, con perfiles de egreso similares que trascienden la experiencia de solo cursar un semestre en alguna de las instituciones.

Conjuntamente con lo anterior, socializar esta experiencia es también una invitación para que otras escuelas se puedan sumar en el diseño de propuestas colectivas de formación incidentes para el continente, potenciando la apuesta sostenida de un diálogo que, como ethos cultural, oriente la relación entre la academia y la sociedad.

\section{REFERENCIAS BIBLIOGRÁFICAS}

Aupetit, S. D. (12 de septiembre de 2018). https:// es.scribd.com/. Obtenido de https://es.scribd.com/document/95713506/La-Internacionalizacion-de-la-Educacion-Superior-en-America-Latina-Oportunidades-y-Desafios

Bauman, Z. (2004) Modernidad Líquida: Fondo de la Cultura Económica de Argentina S.A. Buenos Aires.

Brunner, J. \& Miranda, A. Educación Superior Iberoamericana, 2016. Cinda, Universia.RIL editores, Santiago de Chile, consultado en: https://cinda.cl/publicacion archivos/ educacion-superior-en-iberoamerica-informe-2016/

Declaración de Bolonia (1999). Declaración de los Ministros Europeos de Educación.

Didou Aupetit, S. (2014) La UNESCO y la educación superior, 2014-2017: aportes de la Reunión de Cátedras UNESCO sobre la educación superior, las TIC en la educación y los profesores. Consultado el 29 de enero de 2018, disponible en http://www.unesco.org/new/fileadmin/MULTIMEDIA/HQ/ED/pdf/UNESCO-summary-report-chairs-2014-1. pdf
Gairín, J., \& Rodríguez - Gómez, D. (2011). Presentación. Educar., 9 - 12. Consultado en http://www.redalyc.org/ articulo.oa?id=342130836001

Jara, O. (2011). Orientaciones teórico-prácticas para la sistematización de experiencias. San José de Costa Rica: Alforja. Obtenido de http://www.bibliotecavirtual.info/ wp-content/uploads/2013/08/Orientaciones_teorico-practicas_para_sistematizar_experiencias.pdf

Mora J. (2004). La Necesidad del Cambio Educativo para la Sociedad del Conocimiento. Rev. Iberoamericana de Educación $\mathrm{N}^{\circ}$ 35. 13- 37

Roca, E. (2010). Cambio del Modelo Educativo. Revista del Centro de Estudios Jordi Puyol, 92 - 118.

Rozas, M. (2004). La intervención social en relación con la cuestión social. Buenos Aires, Argentina: Espacio Editorial.

Ruiz, L. (13 de septiembre de 2001). La sistematización de Prácticas. Obtenido de https://www.bing.com/search?q=la+sistematizaci $\%$ C 3\%B3n+de+pr\%C3\%A1cticas\%$2 \mathrm{C}+$ Ruiz+L.\&form=IENTHT\&mkt=es-cl\&httpsmsn=1\&refig $=73 c 2$ fa5839eb466a9caa8e0b0ddc90a0\&sp=-1\&p$q=$ la+sistematizaci $\% C 3 \% B 3 n+d e+p r \& s c=0-24 \& q s=n \& s-$ $\mathrm{k}=\& \mathrm{cvid}=73 \mathrm{c}$ fa5839eb466a9caa8e0b0ddc90a0

Salas, R. (2008) Conocer desde el sur. Para una cultura política emancipatoria. Boaventura de Sousa Santos. Polis, Revista de la Universidad Bolivariana V. 7. N 21. Editorial Universidad Bolivariana, Santiago, pp. 415-419. 285.

Schön, D. (2010). La Formación de profesionales reflexivos. Hacia un nuevo Diseñado de la Enseñanza y el Aprendizaje en las en las Profesiones. Barcelona: Paidós.

Travi, B. 2014. Investigación histórica e identidad en trabajo social. Nuevas y renovadas epistemologías para los nuevos tiempos. Revista del Departamento de Ciencias Sociales, 5:37-58

Universidad Católica Silva Henríquez, Internacionalización. Consultado el septiembre 2018, disponible en http:// ww3.ucsh.cl/vinculacion-con-el-medio

Universidad Católica Silva Henríquez (2012). Plan de Desarrollo Estratégico 2012 - 2020. Santiago de Chile. UCSH.

Waldman Mitnick, G. (2000). Los rumbos de la educación superior: tendencias y desafíos. Revista Mexicana de Ciencias Políticas y Sociales [en línea] 2000, XLIV (septiembre-diciembre) [Fecha de consulta: 29 de enero de 2019] Disponible en:<http://www.redalyc.org/articulo. oa?id=42118016> ISSN 0185-1918. 